\title{
Demonstration of Space Optical Transmitter Development For Multiple High Frequency Bands
}

\author{
Hung Nguyen, Rainee Simons, Edwin Wintucky, and Jon Freeman \\ NASA Glenn Research Center \\ 21000 Brookpark Road, MS:54-5 \\ Cleveland, Ohio 44135
}

As the demand for multiple radio frequency carrier bands continues to grow in space communication systems, the design of a cost-effective compact optical transmitter that is capable of transmitting selective multiple RF bands is of great interest, particularly for NASA Space Communications Network Programs. This paper presents experimental results that demonstrate the feasibility of a concept based on an optical wavelength division multiplexing (WDM) technique that enables multiple microwave bands with different modulation formats and bandwidths to be combined and transmitted all in one unit, resulting in many benefits to space communication systems including reduced size, weight and complexity with corresponding savings in cost. Experimental results will be presented including the individual received RF signal power spectra for the $L, C, X$, $\mathrm{Ku}, \mathrm{Ka}$, and $\mathrm{Q}$ frequency bands, and measurements of the phase noise associated with each RF frequency. Also to be presented is a swept RF frequency power spectrum showing simultaneous multiple RF frequency bands transmission. The RF frequency bands in this experiment are among those most commonly used in NASA space environment communications.

\section{Introduction}

There is often expressed the need to improve on transporting multiple frequency carriers over a long distance in free-space [1,2]. One effective way is to transport multiple RF frequency carriers through a common optical beam, which is due to the fact that a hybrid RF/optical system can provide a very high data capacity signal communication as well as significantly reduce power, volume and complexity as opposed to RF infrastructure system alone. Traditionally, the various frequency bands used for spacecraft navigation and communications are transmitted by means of a separate transmitter terminal for each frequency. Based on an optical wavelength division multiplexing (WDM) technique, which is generated by tunable diode laser 
(TDL), the transmitting system enables multiple microwave bands to be combined and transmitted all in one transmitter unit as shown in Figure 1.

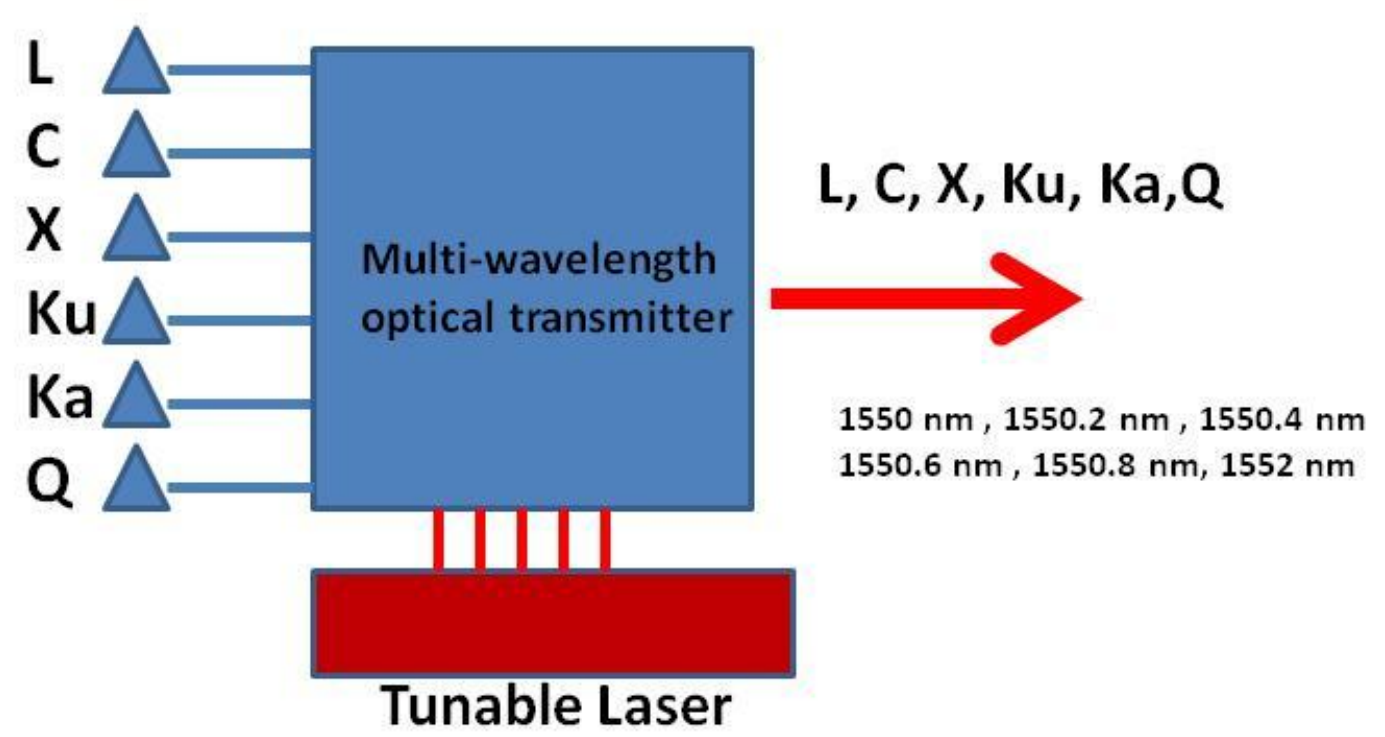

Figure 1: WDM/TDL Technique of Selective RF Carriers

The operating principle is based on the use of the WDM technique for transmitting multiple high frequency microwave bands. The WDM technique uses a different optical wavelength to carry each separate and independent high-frequency microwave band (e.g., L, C, X, Ku, Ka ,Q or higher bands). Though the use of WDM to combine the RF multiple frequency bands has been studied previously [2,3,4], it still faced many difficult challenges. This is because of serious problem s connected with the crosstalk that can arise from inter-channel interference in a WDM system. The crosstalk resulting from adjacent RF channel interference depends upon several factors such as the channel spacing, locking signal level, and the line width enhancement factor of the laser incorporated in the design of the optical discriminator. Recently, because of the many improvements in multiplexing systems and tunable laser semiconductor diodes [5,6,7], such problems are becoming less of an issue. Since each RF carrier operates at a different optical wavelength, minimal cross-talk among RF carriers can be achieved with the use of a tunable diode laser. The spacing wavelength can be adjusted through an electronic tunable laser controller unit and can thereby minimize the crosstalk effect.

The implementation of WDM using a tunable laser can result in a single transmitter terminal unit that that serves multiple RF frequency bands rather and eliminates the need to use a separate terminal for each frequency. Additionally, the flexibility of the tunable laser concept is another important asset because additional RF carriers can be 
added into the system at anytime with minimal configuration. Tangible benefits to communication systems shown in Figure 2 include significant reductions in the communication satellite payload power and weight requirements with corresponding significant reductions in costs. Figure 2 illustrates the multiple frequency carriers that can be transported optically on a single beam among communication systems on satellites and earth stations instead of RF domain distribution. In traditional RF communication systems, to accommodate the multiple frequency carriers, the communication system requires additional transceivers and a number of antennas, which can result in high operation, maintenance and reconfiguration costs [8].

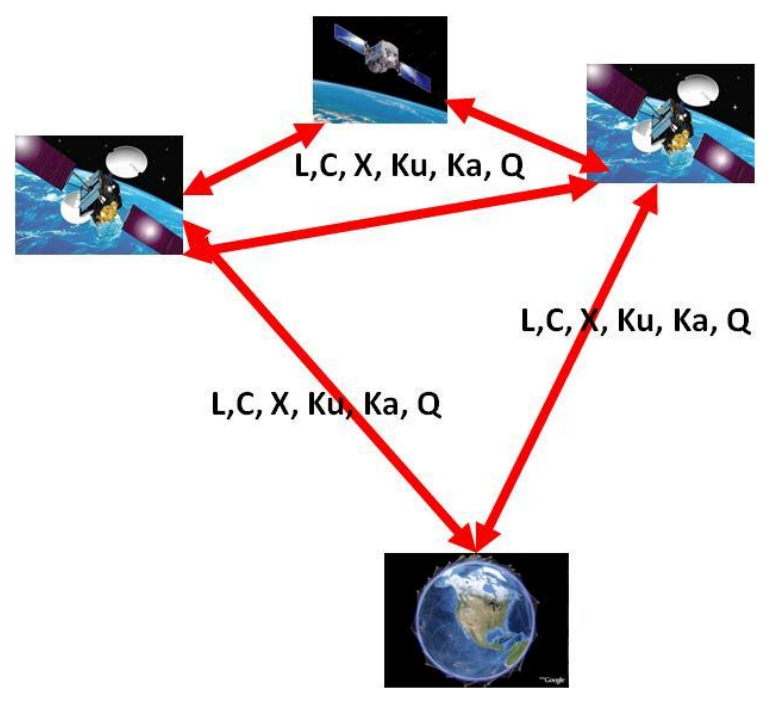

Figure 2: Illustration of Distribution Multiple Frequency Carriers on Satellites and Earth Stations

\section{Experiment and Results}

Our setup experiment for demonstrating an optical transmitter for multiple radio frequency bands, shown in Figure 3, utilizes the tunable diode laser (TDL) and electro optic (EO) modulator to modulate each RF carrier. In order to achieve the modulated optical carrier, the approach was to use the EO modulator to combine the RF carriers with the optical carrier using the WDM technique. In this scenario, each of the RF carriers is assigned to a different optical wavelength. The modulated optical signals were added together by using a multiplexer before sending out to free space or optical fiber. The wavelength separation among these optical frequencies was set to $2 \mathrm{~nm}$ to assure the minimal crosstalk at the receiver end. 
In Figure 3, the hybrid RF/optical transmitter was setup to include six RF carrier frequencies $(1.5,7,8.4,14.5,32$, and $38 \mathrm{GHz})$, each of which were modulated at six different optical wavelengths (1550, 1550.2, 1550.4, 1550.6, 1550.8, and $1552 \mathrm{~nm}$ ) generated by the tunable laser. The tunable laser has a $50 \mathrm{~ms}$ line width and its tunable wavelength ranged between 1520 and $1570 \mathrm{~nm}$ with the averaged optical power measured at $21 \mathrm{~mW}$.

Each RF carrier was then used to drive the electro-optic modulator (EOM), which was capable of modulating RF frequencies up to $40 \mathrm{GHz}$. The average insertion loss of these EO modulations was $3.4 \mathrm{~dB}$ in the frequency range of 1 to $40 \mathrm{GHz}$. The EOM was externally DC biased at about $5.2 \mathrm{~V}$, which was the modulation voltage needed for achieving linear operation. The electrical RF power applied to the EOM was $16 \mathrm{dBm}$ for all the carrier frequencies under test in our experiment. The down-converted optical signal was then filtered and detected by a high speed photo-detector (PD). The PD had a response of $0.4 \mathrm{~A} / \mathrm{W}$, over its operating wavelength range of $950-1650 \mathrm{~nm}$ with an output impedance of $50 \Omega$.

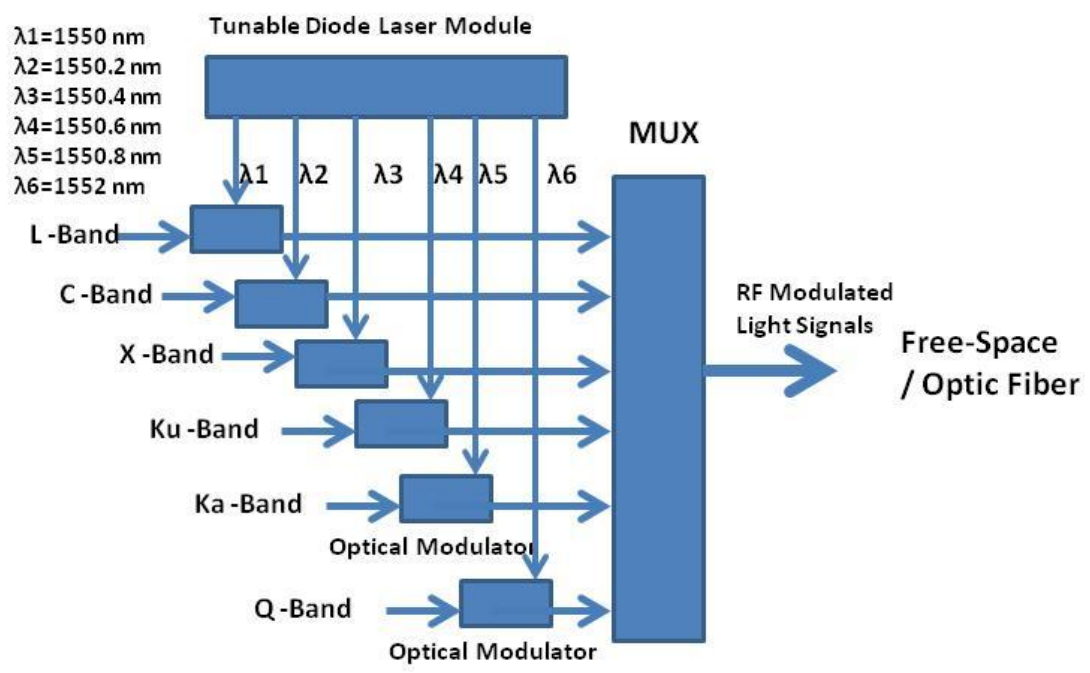

Figure 3: Experiment Setup for Demonstration of Multiple RF Carriers

The performance of this multi-wavelength optical transmitter was evaluated from both the RF power spectrum and phase noise corresponding to each frequency band. The electrical RF power and phase noise for each RF carrier frequency were measured by using an RF spectrum analyzer. Figure 4(A) through 4(F) show the measured RF power at the output of the PD after down-conversion. As shown in these figures, the RF power loss between the $L$ and Ka band was observed to be $18 \mathrm{~dB}$. This implies that the EOM used for modulating RF carriers was attenuated in the higher frequency range. From the frequency response of the EOM used in our experiment, there was a $7.4 \mathrm{~dB}$ loss 
starting from $1 \mathrm{GHz}$ to $40 \mathrm{GHz}$ due to saturation effects. The results indicate that the transmitter system when employing a low insertion loss EO modulator can support many RF carriers while still maintaining a high data bandwidth. A swept frequency power spectrum showing simultaneous $C$ and $X$ frequency band transmission is illustrated in Figure 5. Only two frequency bands were validated at this time due to limitations of our equipment; however, more than two frequency bands can be achieved with this method. It is clear from this result that the difference in the power spectrum of $2.8 \mathrm{~dB}$ is due to the loss attributed mainly to the EOM.

The detailed aspects of the phase noise for each of the frequency bands are shown in Figure 6(A) through 6(D), exhibiting a degradation of an average $9.5 \mathrm{dBc} / \mathrm{Hz}$ at $10 \mathrm{KHz}$ over these frequency ranges. From these results, it seems that the shot noise and thermal noise generated from the PD and tunable lasers contribute to this degradation of the phase stability. The average phase noise for different RF bands exhibits slight variations as shown in Figure 7 . It is observed that since the EO modulations have the low saturated state at the lower frequency band, the degradation of RF phase noise seems to occur at the low frequency region. In addition, as the input frequency to the EO modulator increases, the phase noise tends to decrease linearly.

\section{Conclusion}

We successfully demonstrated the feasibility of a multi-wavelength optical transmitting technique (WDM) for delivering selective multiple radio frequency bands such as $\mathrm{L}, \mathrm{C}$, $\mathrm{X}, \mathrm{Ku}, \mathrm{Ka}$, and $\mathrm{Q}$ bands utilizing a tunable diode laser/EOM for potential satellite communication applications. Significant reduction in volume, weight, and power can be realized by using this technique; however, the stability of tunable laser is essential to minimize the cross-talk among the RF carriers. The results show that the RF power outputs for multiple frequency bands show good performance using tunable laser concept combined with electro-optic modulations. The RF phase noise shows slight variations as the frequency bands varies. Thus, it can be concluded that this concept can improve the system flexibility and scalability for both ground and space communication applications. 
A

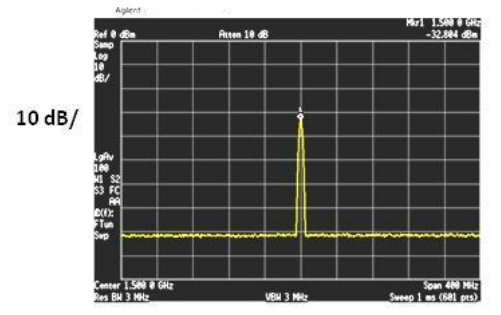

$L-B$ and $(1.5 \mathrm{GHz})$ at $1550 \mathrm{~nm}$

Start: $1.3 \mathrm{GHz}$

Stop: $1.7 \mathrm{GHz}$

Span: $400 \mathrm{MHz}$

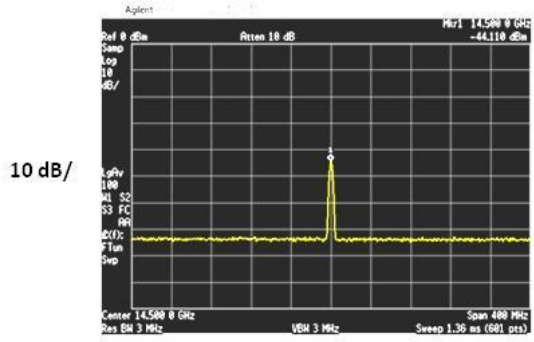

Ku-Band $(14.5 \mathrm{GHz})$ at $1550.6 \mathrm{~nm}$

Start: $14.3 \mathrm{GHz}$

Stop: $14.7 \mathrm{GHz}$

Span: $400 \mathrm{MHz}$
B

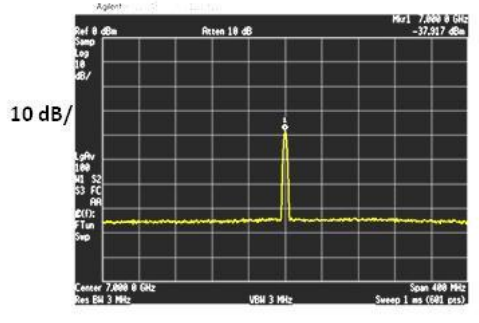

C-Band $(7 \mathrm{GHz})$ at $1550.2 \mathrm{~nm}$

Start: $6.8 \mathrm{GHz}$

Stop: $7.2 \mathrm{GHz}$

Span: $400 \mathrm{MHz}$

E

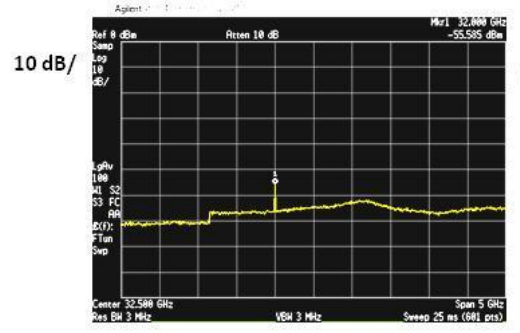

$\mathrm{Ka}-\mathrm{B}$ and $(32 \mathrm{GHz})$ at $1550.8 \mathrm{~nm}$

Start: $30 \mathrm{GHz}$

Stop: $35 \mathrm{GHz}$

Span: $900 \mathrm{MHz}$

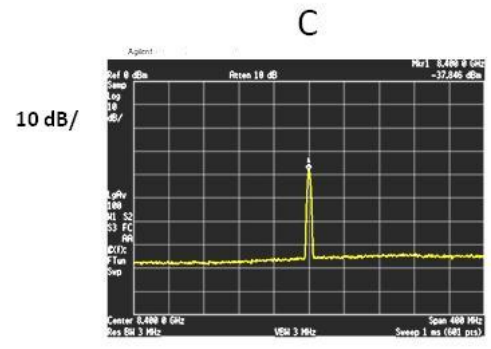

$X-B$ and $(8.4 \mathrm{GHz})$ at $1550.4 \mathrm{~nm}$ Start: $8.2 \mathrm{GHz}$

Stop: $8.6 \mathrm{GHz}$ Span: $400 \mathrm{MHz}$

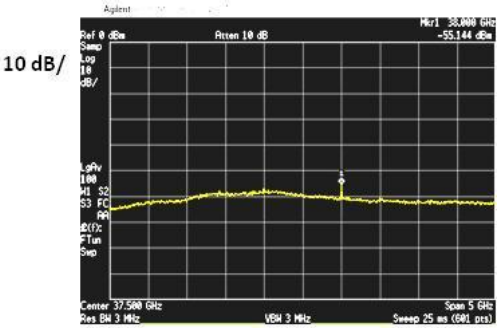

Q-Band $(38 \mathrm{GHz})$ at $1552 \mathrm{~nm}$ Start: $35 \mathrm{GHz}$ Stop: $40 \mathrm{GHz}$

Span: $900 \mathrm{MHz}$

Figure 4 (A - F): RF Power Output Performance of L, C, X, Ku, Ka and Q Bands.

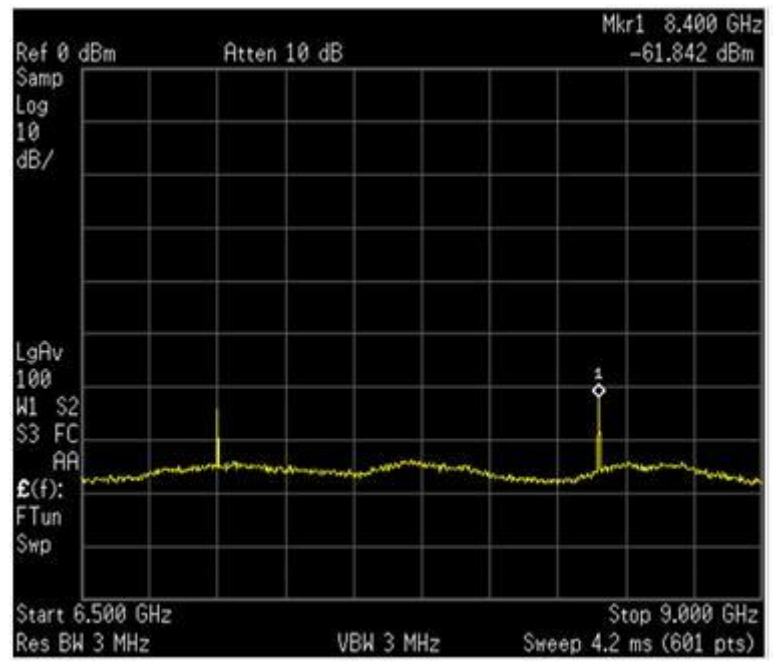

Figure 5 : RF Power Output Performance of simultaneous $C$ and $X$ bands. 
A

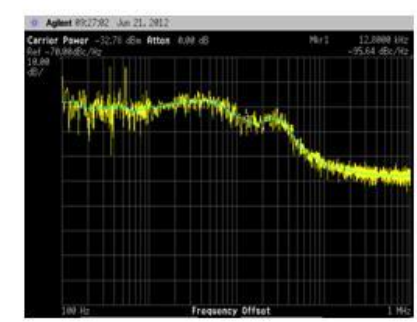

L-Band (1.5 GHz) at $1550 \mathrm{~nm}$
B

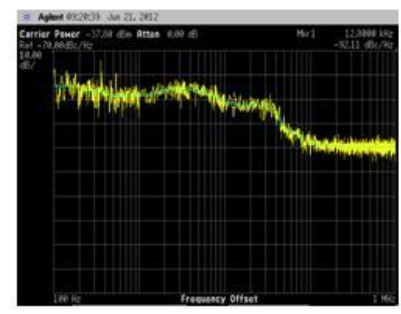

C-Band (7 GHz) at $1550 \mathrm{~nm}$

D

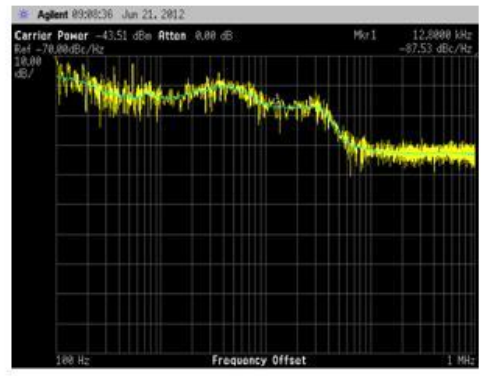

Ku-Band $(14.5 \mathrm{GHz})$ at $1550 \mathrm{~nm}$

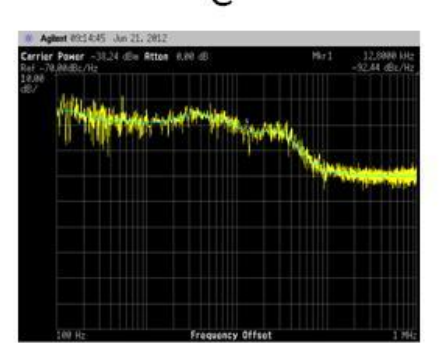

X-Band $(8.4 \mathrm{GHz})$ at $1550 \mathrm{~nm}$

Figure 6 (A - D): Phase Noise Output Performance of L, C, X, and Ku Bands.

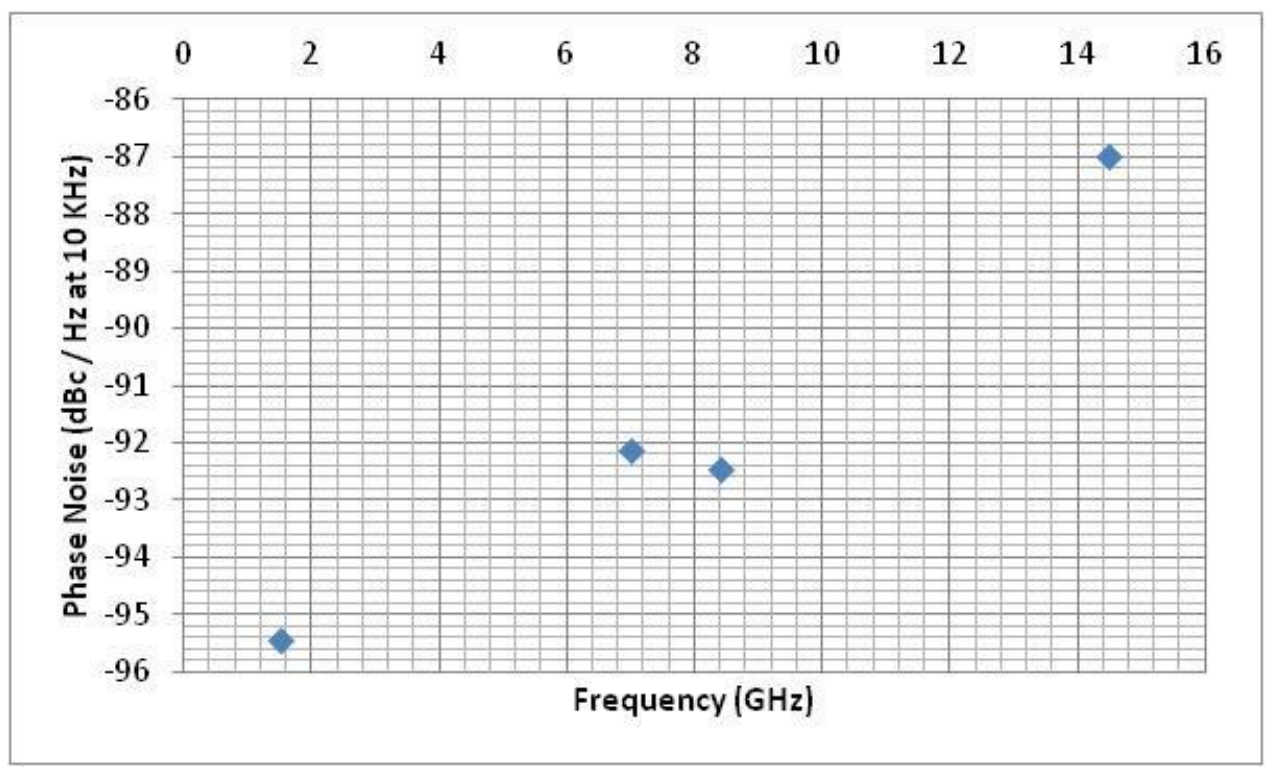

Figure 7: Average Phase Noise Output Performance of L, C, X, and Ku Bands. 


\section{References}

[1] M. L. Dennis, J. A. Nanzer, P. T. Callahan, M. C. Gross, T. R. Clark,D. Novak, and R. B. Waterhouse, "Photonic upconversion of $60 \mathrm{GHz}$ IEEE 802.15.3c standard compliant data signals using a dual-wavelength laser," in Proc. 23rd Annu. Meeting IEEE Photon. Soc., 2010.

[2] Slaveski, F., Sluss, J.,Jr., Atiquzzaman, M.,Nguyen, H., and Ngo, D., (2003), Optical Fiber Wavelength Division Multiplexing, IEEE Aerospace and Electronic Systems Magazine.

[3] Hoon Kim," Radio over RSOA-based WDM-PON," Microwave Photonics, 2011 International Microwave Photonics Conference, 2011 Asia-Pacific, MWP/APM.

[4] Kenji Miyamoto, et al.,"Experimental Demonstration of MIMO RF Signal Transmission in RoF-DAS over WDM-PON," Microwave Photonics, 2011 International Microwave Photonics Conference, 2011 Asia-Pacific, MWP/APM.

[5] San-Liang Lee, et al.," Integration of Semiconductor Laser Amplifiers with Sampled Grating Tunable Lasers for WDM Applications," IEEE Journal of Selected Topics in Quantum Electronics, Vol. 3, No.2, April 1997.

[6] Domanic Lavery, et al., "Demonstration of $10 \mathrm{Gbit} / \mathrm{s}$ Colorless Coherent PON Incorporating Tunable DS-DBR Lasers and Low-Complexity Parallel DSP OFC/NFOEC Postdeadline Papers 2012 OSA.

[7] C. H. Yeh, et al., "Wavelength-Tunable Laser for Signal Remodulation in WDM Access Networks Using DPSK Downlink and OOK Uplink," IEEE Photonics Technology Letters, Vol. 21, No. 22, November 15, 2009.

[8] Mark L. Psiaki, et al., "Design and Practical Implementation of Multifrequency RF Front Ends Using Direct RF Sampling," IEEE Transactions on Microwave Theory and Techniques, Vol. 53, No. 10, October 2005. 